\title{
Development and tuning of bacteria foraging optimization algorithm on cell formation in cellular manufacturing system
}

\begin{abstract}
Ever since Kevin M. Passino invented the bacteria foraging optimization algorithm, one of the main challenges has been employment of the algorithm to problem areas other than those for which the algorithm was proposed. This research work inquires the applications of designed experiments aided by multiple regression analysis for tuning of this emerging novel optimization algorithm parameters to the cell formation $(\mathrm{CF})$ problem considering operation sequence. In this paper, an attempt is made to tuning chemo tactic and swarming steps parameters meanwhile taking into consideration bacteria foraging optimization algorithm convergence speed and performance. The factorial designed experiment is suggested to create treatments of experiment. The adequacy of the proposed model is analyzed based on some commonly statistical criteria. The results lie in favor of adequacy of the proposed model.
\end{abstract}

Keyword: Cell formation; Bacteria foraging optimization algorithm; Designed experiments; Cellular manufacturing system 